\title{
Efecto del movimiento de contenedores sobre la eficiencia de las principales economías operadoras de carga del APEC
}

\author{
Effect of Container Movement on the Efficiency \\ of the main APEC Cargo Operating Economies
}

DOI: $10.32870 /$ mycp.v10i30.739

\author{
Osvaldo Urbano Becerril-Torres ${ }^{1}$ \\ Gabriela Munguía-Vázquez ${ }^{2}$ \\ Rosa María Nava-Rogel ${ }^{3}$
}

\begin{abstract}
Resumen
La Cuenca del Pacífico es una región que se caracteriza por su dinamismo económico. En ella se localizan algunas de las economías más importantes del mundo que integran asociaciones y acuerdos comerciales relevantes, entre ellos el Foro de Cooperación Económica de Asia-Pacífico (APEC, por sus siglas en inglés). Un elemento que distingue al APEC, es que muchas de las economías que lo integran cuentan con fuertes infraestructuras portuarias y un movimiento importante de mercancías mediante contenedores, lo que puede incidir en su eficiencia. Esto les aporta una ventaja en el comercio marítimo. Por ello, el objetivo de este artículo es analizar si el movimiento de contenedores contribuye a mejorar la eficiencia técnica del APEC. Para el análisis se emplea la metodología de Stochastic Frontier Analysis. Entre los resultados destacan que las principales economías operadoras de carga de contenedores, en general, mejoran su eficiencia, aun cuando también se detecta que existe un marginal proceso de divergencia en ésta.
\end{abstract}

Palabras clave: eficiencia técnica, fronteras estocásticas, APEC, infraestructura portuaria, TEU.

\begin{abstract}
The Pacific Basin is a region characterized by its economic dynamism. In it, some of the most important economies in the world that integrate relevant trade associations and agreements are located, including the Asia-Pacific Economic Cooperation Forum. One element that distinguishes it is that many of the economies that comprise it have strong port infrastructures and a significant movement of goods through containers, which can affect its efficiency. This gives them an advantage in maritime trade. Therefore, the objective of this work is to analyze if the movement of containers contributes to improving the technical efficiency of APEC. Stochastic Frontier Analysis methodology is used for the analysis. The results include that the main container cargo operating economies, in general, improve their efficiency, even though it is also detected that there is a marginal process of divergence in this.
\end{abstract}

Keywords: technical efficiency, stochastic frontiers, APEC, port infrastructure, TEUs.

Artículo recibido el 09 de diciembre de 2020 y dictaminado el 10 de marzo de 2021.

1. Universidad Autónoma del Estado de México, Facultad de Economía. Cerro de Coatepec, s/n. Ciudad Universitaria. Toluca, Estado de México, México. C. P. 50110. ORcid: https://orcid.org/0000-00025685-5636. Correo electrónico: obecerrilt@uaemex.mx

2. Universidad Autónoma del Estado de México, Facultad de Economía. Cerro de Coatepec, s/n. Ciudad Universitaria. Toluca, Estado de México, México. C. P. 50110. ORcid: https://orcid.org/0000-00023753-8141. Correo electrónico: gmunguia2000@hotmail.com

3. Universidad Autónoma del Estado de México, Facultad de Contaduría y Administración. Cerro de Coatepec, s/n. Ciudad Universitaria. Toluca, Estado de México, México. C. P. 50110. OrciD: https:// orcid.org/0000-0003-2611-3903. Correo electrónico: rosanr06@yahoo.com.mx 


\section{Introducción}

La Cuenca del Pacífico es escenario de múltiples estudios por la relevancia que tiene para el mundo. Algunas de las economías más importantes se encuentran en esta región, como la china, la japonesa y la estadounidense. De manera particular, la región del Foro de Cooperación Económica de Asia-Pacífico (APEC, por sus siglas en inglés) incluye a las economías más influyentes de la zona. En ésta se encuentran muchos de los puertos más importantes a nivel global, tanto importadores como exportadores de carga en contenedores, así como también algunas de las principales rutas marítimas con mayor movimiento de contenedores. Basta decir que en el año 2018, datos del World Shipping Council (2020a) reportan que el puerto de Shanghái movió un volumen de 42.01 millones de contenedores, mientras que Singapur y Shenzhen desplazaron 36.6 y 27.74 millones, respectivamente, siendo estos tres puertos los principales en el mundo.

Por su relevancia global, la región Asia-Pacífico despierta el interés como objeto de estudio en las diferentes áreas del conocimiento científico. Entre ellas, temas relacionados con el medio ambiente son abordados por Xu et al. (2019), Ansari et al. (2019), Anees et al. (2019), Zhou et al. (2019) y Wasif et al. (2019). Los temas relacionados con la salud mental son considerados por Chiang et al. (2019) y por Dowrick et al. (2020). O con el sector externo, son relevantes los estudios de Erkekoğlu y Yilmaz (2019). Son también de interés temáticas vinculadas con la construcción de escenarios futuros, las cuales son estudiadas por Zhang et al. (2020) y Sun et al. (2019), o investigaciones en relación con la rivalidad económica regional son abordadas por Chaekwang (2019).

En particular, por su importancia la región del APEC ha sido objeto de diversos estudios relacionados con la eficiencia y productividad. En los últimos 15 años se pueden encontrar algunos relacionados con la productividad y el crecimiento económico (Wu, 2004); con ahorro energético y su eficiencia (Jin-Li \& Chih-Hung, 2007); con la eficiencia del transporte (APEC Secretariat, 2008); con el sector agropecuario (Ayvar et al., 2018; Yu et al., 2011); con las telecomunicaciones (Chun-Hsiung \& Chun-Yu, 2012); con los puertos y las aduanas (Delfin \& Navarro, 2017; Infante \& Gutiérrez, 2013; Wu et al., 2016; Zamora, 2017), o incluso con el medio ambiente (Wang et al., 2016).

Sin embargo, no se ha encontrado algún estudio que permita observar el efecto que tiene el movimiento de contenedores en la eficiencia técnica de 
las economías del APEC, no obstante la relevancia para sus puertos, siendo así que éste se constituye como una variable de interés, para ser considerada en la función de producción de estas economías, como lo reconoce la United Nations Conference on Trade and Development (UNCTAD, 2019). Derivado de esto, surge la siguiente interrogante: ¿el movimiento de contenedores en los puertos de las economías del APEC tiene algún efecto sobre el uso de los factores, es decir, sobre su eficiencia técnica?, a lo que hipotéticamente se plantea que sí existe un efecto favorecedor, dado que ello impulsa y es estimulado por el afán de hacer más competitivas a las economías en el escenario geoeconómico regional.

Por lo anterior, este análisis se aborda en el presente documento con el objeto de contribuir analíticamente al entendimiento de la región del APEC desde esta perspectiva. De ello, el objetivo de esta investigación es analizar el efecto que tiene el movimiento de contenedores en la eficiencia técnica de las economías del APEC, e identificar cuáles de ellas se ven más favorecidas y cuáles lo hacen en menor medida.

En este contexto de estudio, es reconocido por la UNCTAD (2019) que el análisis del transporte marítimo es complejo, dado su carácter internacional y su dimensionalidad multilateral. Derivado de esto, plantea características particulares en su análisis, ya que puede ser considerado, entre otros aspectos, como un factor productivo más para otros sectores y ámbitos de la actividad económica, como el comercio, la pesca, el turismo y la energía. Derivado de ello, la posición que ocupa una región, país o un puerto en el entramado global de la red de transporte marítimo de contenedores, id est, su conectividad, es un importante elemento para la accesibilidad al comercio mundial, los costos del comercio y la competitividad, así como la productividad y la eficiencia, entre otros.

Así, para estimar la eficiencia técnica se emplea el enfoque teórico del Análisis de Fronteras Estocásticas (Stochastic Frontier Analysis), siguiendo la propuesta metodológica de Battese y Coelli (1995), para un panel de datos, constituido por las economías del APEC que se encuentran en la lista de los 30 principales operadores de contenedores en el mundo, a través del periodo temporal 2010-2018.

Derivado de lo anterior, y para alcanzar el objetivo, el documento se estructura de la siguiente manera: en el apartado dos se presentan algunas características de la región Asia-Pacífico, centrando la atención en la región del APEC en relación con el movimiento de contenedores. En la sección tres se 
realiza una revisión de la literatura existente sobre el análisis de la eficiencia y la productividad y sus contextos de estudio, en donde se ha identificado la carencia de estudios sobre el efecto del movimiento de contenedores sobre la eficiencia técnica de las economías del APEC. En la cuarta sección se presenta la metodología a utilizar para la estimación de la eficiencia técnica de las economías objeto de estudio. En las secciones cinco y seis se hace referencia a los datos y fuentes de información empleados para la estimación de la eficiencia, así como a los resultados obtenidos, respectivamente, y, por último, se presentan las conclusiones a partir de los resultados del estudio.

\section{Características de la región Asia-Pacífico}

La región Asia-Pacífico es una de las más dinámicas en el movimiento de mercancías por vía marítima. En ésta se localizan algunas de las economías más dinámicas del mundo, así como aquellas que son líderes en el desplazamiento de mercancías en contenedores. En esta región se encuentran también diversas organizaciones y acuerdos multilaterales más recientes e importantes del mundo, como el Comprehensive and Progressive Agreement for TransPacific Partnership, conocido como TPP-11. Uno de los más destacados es el Asia-Pacific Economic Cooperation (APEC), integrado por 21 economías de la región, y cuyo principal objetivo es facilitar el crecimiento económico, la cooperación técnica y económica, la facilitación y liberalización del comercio y las inversiones en la región.

En este contexto, la UNCTAD (2018) reconoce que el tráfico portuario de carga es una función de la evolución de la economía y la demanda global, particularmente de la producción, de la inversión y de las necesidades de consumo. La UNCTAD estima que el tráfico portuario mundial de contenedores aumentó un 6\% en 2017, triplicando el valor del año 2016. Según sus datos, el volumen manipulado en los puertos de contenedores ascendió a 752.2 millones de twenty foot equivalent unit (TEU) ${ }^{4}$ en 2017.

De acuerdo con la UNCTAD (2018), entre los principales factores del aumento de volumen destacan el fuerte crecimiento registrado en la ruta comercial entre los

4. Ésta es una unidad de medida de capacidad del transporte marítimo, empleada para terminales portuarias, así como para contenedores y para buques portacontenedores. Ésta es expresada de manera específica en contenedores. Una TEU es la capacidad de carga de un contenedor normalizado de 20 pies. 
países asiáticos, una mayor demanda de consumo en Estados Unidos y Europa, y el aumento del comercio Norte-Sur. En particular Asia desempeña un papel fundamental en el comercio y el transporte marítimo mundial. En la región Asia-Pacífico — según datos de la UNCTAD (2018) - se concentran cerca del $42 \%$ de los puertos. En este escenario, Asia domina la actividad de manipulación de contenedores con el $65 \%$ del tráfico portuario, como se muestra en el figura 1.

\section{Figura 1}

Tráfico portuario de contenedores en el mundo, por regiones, 2017 (\% de TEU totales)



Fuente: elaboración de los autores con datos de UNCTAD (2018).

En el mundo, las principales rutas marítimas comerciales se encuentran en la región Asia-Pacífico, siendo la Asia-América del Norte, con dirección hacia el Oeste, la que menor movimiento de contenedores tuvo en el año 2017, con 7'490,000 contenedores de 20 pies, en tanto que hacia el Este el movimiento fue de 19'482,000 de contenedores. Le siguió la ruta Asia-Norte de Europa, con 9'924,000 hacia el Oeste, y hacia el Este 5'139,000; y la AsiaMediterráneo, con 5’504,000 en dirección Oeste y 2'409,000 hacia el Este, como se observa en la tabla 1. 
Tabla 1

Principales rutas de comercio marítimo en el mundo

\begin{tabular}{|c|c|c|c|c|c|}
\hline \multicolumn{6}{|c|}{ Principales rutas comerciales (TEU enviados) 2017} \\
\hline Ruta & Hacia el Oeste & Hacia el Este & Hacia el Norte & Hacia el Sur & Total \\
\hline $\begin{array}{l}\text { Asia-América del } \\
\text { Norte }\end{array}$ & $7 ’ 490,000$ & $19^{\prime} 482,000$ & ------- & ------- & $26{ }^{\prime} 572,000$ \\
\hline $\begin{array}{l}\text { Asia-Norte de } \\
\text { Europa }\end{array}$ & 9'924,000 & $5 ’ 139,000$ & ------- & ------- & $15^{\prime} 063,000$ \\
\hline $\begin{array}{l}\text { Asia- } \\
\text { Mediterráneo }\end{array}$ & $5 ’ 504,000$ & $2 ’ 409,000$ & ------- & ------- & 7’913,000 \\
\hline $\begin{array}{l}\text { Asia-Medio } \\
\text { Oriente }\end{array}$ & 3’340,000 & $1^{\prime} 400,000$ & ------ & ------ & ------ \\
\hline $\begin{array}{l}\text { Norte de Europa- } \\
\text { Norteamérica }\end{array}$ & $3 ’ 284,000$ & $2 ' 120,000$ & ------- & ------- & $5^{\prime} 404,000$ \\
\hline $\begin{array}{l}\text { Asia-Costa este } \\
\text { Sudamérica }\end{array}$ & ------ & |------ & 730,000 & 1’344,000 & 2’074,000 \\
\hline $\begin{array}{l}\text { Norte de Europa } \\
\text { / Mediterráneo- } \\
\text { Costa este } \\
\text { Sudamérica }\end{array}$ & ------- & ------- & 830,000 & 850,000 & 1'680,000 \\
\hline $\begin{array}{l}\text { América del } \\
\text { Norte-Costa este } \\
\text { América del Sur }\end{array}$ & ------- & ------- & 794,000 & 474,000 & $1^{\prime} 268,000$ \\
\hline
\end{tabular}

Fuente: World Shipping Council (2020b).

De los 50 principales puertos con movimiento de contenedores del mundo, 35 se encuentran en países del APEC, resaltando así la importancia de la región, como se muestra en la tabla 2, donde Shanghái es el líder con un volumen de 42.01 millones de TEU en el año 2018. Le siguen en importancia el puerto de Singapur con 36.6 millones de TEU y el puerto de Shenzhen con 27.74 millones. 
Tabla 2

Principales 50 puertos de contenedores de Asia-Pacífico 2014-2018

\begin{tabular}{|c|c|c|c|c|c|c|}
\hline Ranking & Puerto & 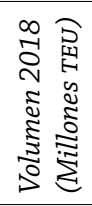 & 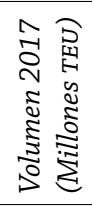 & 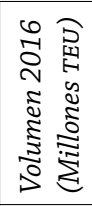 & 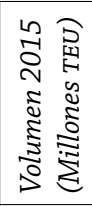 & 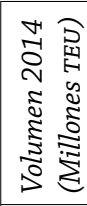 \\
\hline 1 & Shanghái, China & 42.01 & 40.23 & 37.13 & 36.54 & 35.29 \\
\hline 2 & Singapur & 36.60 & 33.67 & 30.90 & 30.92 & 33.87 \\
\hline 3 & Shenzhen, China & 27.74 & 25.21 & 23.97 & 24.20 & 24.03 \\
\hline 4 & Ningbo-Zhoushan, China & 26.35 & 24.61 & 21.60 & 20.63 & 19.45 \\
\hline 5 & Guangzhou Harbor, China & 21.87 & 20.37 & 18.85 & 17.22 & 16.16 \\
\hline 6 & Busan, Corea del Sur & 21.66 & 20.49 & 19.85 & 19.45 & 18.65 \\
\hline 7 & Hong Kong, S.A.R, China & 19.60 & 20.76 & 19.81 & 20.07 & 22.23 \\
\hline 8 & Qingdao, China & 18.26 & 18.30 & 18.01 & 17.47 & 16.62 \\
\hline 9 & Tianjin, China & 16.00 & 15.07 & 14.49 & 14.11 & 14.05 \\
\hline 12 & Port Klang, Malasia & 12.32 & 13.73 & 13.20 & 11.89 & 10.95 \\
\hline 14 & Kaohsiung, Taiwán, China & 10.45 & 10.27 & 10.46 & 10.26 & 10.59 \\
\hline 15 & Xiamen, China & 10.00 & 10.38 & 9.61 & 9.18 & 10.13 \\
\hline 16 & Dalian, China & 9.77 & 9.70 & 9.61 & 9.45 & 10.13 \\
\hline 17 & Los Ángeles, Estados Unidos de América & 9.46 & 9.43 & 8.86 & 8.16 & 8.33 \\
\hline 18 & Tanjung Pelepas, Malasia & 8.96 & 8.38 & 8.28 & 9.10 & 8.50 \\
\hline 20 & Long Beach, Estados Unidos de América & 8.09 & 7.54 & 6.80 & 7.19 & 6.82 \\
\hline \multirow[t]{2}{*}{21} & Laem Chabang, Tailandia & 8.07 & 7.78 & 7.22 & 6.82 & 6.58 \\
\hline & Keihin Ports, Japón* & & 7.98 & 7.61 & 7.52 & 7.85 \\
\hline 22 & Tanjung Priok, Jakarta, Indonesia & 7.64 & 6.09 & 5.51 & 5.20 & 5.77 \\
\hline 23 & $\begin{array}{l}\text { Nueva York-Nueva Jersey, Estados Unidos } \\
\text { de América }\end{array}$ & 7.20 & 6.71 & 6.25 & 6.37 & 5.77 \\
\hline 25 & Yingkou, China & 6.50 & 6.28 & 6.08 & 5.92 & 5.77 \\
\hline \multirow[t]{2}{*}{26} & Ho Chi Minh City, Vietnam & 6.33 & 6.16 & 5.99 & 5.31 & 6.39 \\
\hline & Hanshin Port, Japón** & & 5.22 & 5.02 & 4.93 & 5.32 \\
\hline 28 & Manila, Filipinas & 5.05 & 4.82 & 4.52 & 4.23 & 3.65 \\
\hline 32 & Lianyunguang, China & 4.75 & 4.72 & 4.70 & 5.01 & 5.01 \\
\hline 33 & Tokyo, Japón & 4.57 & 4.50 & 4.25 & -- & -- \\
\hline 35 & Savannah, Estados Unidos de América & 4.35 & 4.05 & 3.64 & 3.74 & 3.35 \\
\hline 38 & Rizhao, China & 4.00 & 3.24 & 3.01 & -- & -- \\
\hline 39 & Colón, Panamá & 3.89 & 3.89 & 3.26 & 3.58 & 3.29 \\
\hline
\end{tabular}




\begin{tabular}{|c|c|c|c|c|c|c|}
\hline Ranking & Puerto & 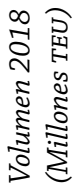 & 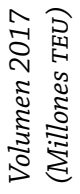 &  & 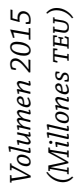 & 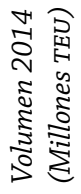 \\
\hline $41^{* * *}$ & $\begin{array}{l}\text { Seattle-Tacoma NW Seaport Alliance, } \\
\text { Estados Unidos de América }\end{array}$ & 3.80 & 3.70 & 3.62 & 3.53 & 3.43 \\
\hline 42 & Dongguan, China & 3.50 & 3.91 & 3.64 & -- & -- \\
\hline 45 & Vancouver, Canadá & 3.40 & 3.25 & 2.92 & -- & -- \\
\hline 47 & Fuzhou, China & 3.34 & 3.01 & 2.66 & -- & -- \\
\hline 49 & Nanjing, China & 3.23 & 3.17 & 3.08 & 2.94 & -- \\
\hline 50 & Cai Mep, Vietnam & 3.20 & 3.07 & 2.56 & -- & -- \\
\hline
\end{tabular}

* Keihin Ports es el centro de superpuertos de Japón en la bahía de Tokio e incluye Yokohama, Kawasaki y Tokio. No hay datos disponibles para 2018 a julio de 2019.

** Hanshin Ports es el centro de superpuertos de Japón en la bahía de Osaka e incluye Kobe, Lsaka, Sakai-Semboku y Amafasaki-Nishinomiya-Ashiya. No hay datos disponibles para 2018 a julio de 2019.

*** En octubre de 2014 el Puerto de Tacoma y el Puerto de Seattle anunciaron un acuerdo para operar de manera conjunta.

Fuente: elaboración de los autores con datos de World Shipping Council (2020a).

Finalmente, esta sección concluye con una reflexión sobre el impacto del Severe acute respiratory syndrome coronavirus 2 , SARS-CoV-2, al sector portuario y movimiento de contendores. Así, la UNCTAD (2020b) identificó que a medida que la pandemia pesaba sobre el comercio marítimo de China, especialmente durante el primer trimestre de 2020, el comercio marítimo mundial estaba destinado a verse afectado. La alta exposición y sensibilidad del sector a los acontecimientos en China, restricciones a los buques y a la tripulación en muchos puertos, la escasez de mano de obra y las restricciones a su movimiento, así como los desafíos operativos que ello implica, han generado un clima de incertidumbre. Los impactos están en todos los ámbitos, que van desde los flujos comerciales marítimos, a los movimientos del barco, cambios de tripulación del barco, capacidad desplegada, operaciones portuarias, capacidad de almacenamiento, hasta conexiones interiores y logística interior.

\section{Estado del objeto de estudio}

Los estudios sobre el uso de los factores de la producción en la Cuenca del Pacífico y en la región del APEC son variados, y difieren en dos sentidos. Por 
un lado, en el análisis de la eficiencia técnica o de la productividad y, por otro, el que conlleva al empleo de dos grandes vertientes metodológicas, identificadas como Análisis de Fronteras Estocásticas (SFA ,por sus siglas en inglés Stochastic Frontier Analysis) y análisis envolvente de datos (DEA, por sus siglas en inglés Data Envelopment Analysis).

Los estudios que analizan la eficiencia, en la mayoría de los casos se realizan en el sentido de Farrell (1957), en tanto que los que estudian la productividad lo hacen a partir de números índices, particularmente del de Malmquist (1953) o alguna variante de éste. El ámbito de estudio es variado, desde aquellos que buscan identificar los orígenes de la productividad y crecimiento en el APEC, pasando por temas de eficiencia energética, en telecomunicaciones, o de la tierra agrícola, hasta temáticas como la portuaria, las aduanas, las terminales de contenedores, o de emisiones de $\mathrm{CO}_{2}$, como se puede observar a continuación.

Hallar los orígenes de la productividad y crecimiento económico y el efecto que sobre ellos tiene la apertura económica es el interés de Wu (2004), quien analiza el impacto de la apertura sobre las fuentes de productividad y crecimiento en las economías del APEC. Específicamente, se emplea una combinación entre las propuestas metodológicas de Huang y Liu (1994) y de Battese y Coelli (1995) para su análisis.

El ahorro energético y su eficiencia es una prioridad para el mundo, y este tema es abordado por Jin-Li y Chih-Hung (2007), quienes analizan el ahorro de energía de 17 economías del APEC durante la última década del siglo xx, mediante el Data Envelopment Analysis. Entre sus resultados encuentran que la eficiencia energética se incrementa generalmente en las economías del APEC.

Al interior del APEC, su secretaría realizó un estudio sobre la eficiencia del transporte. Así, APEC Secretariat (2008) explora la gama de políticas y programas disponibles para los tomadores de decisiones del sector público, para maximizar la eficiencia del sector del transporte en sus jurisdicciones. Para su estudio, la eficiencia energética del transporte considera enfoques que reducen la cantidad de combustibles fósiles utilizados por vehículo/kilómetro recorrido por carretera o ferrocarril, o que reducen los vehículos/kilómetros recorridos en general.

En los orígenes del análisis de la eficiencia técnica, los primeros estudios se realizaron en relación con el sector agropecuario; es así que autores como Yu et al. (2011) regresan a esta temática primigenia y analizan la eficiencia del uso de la tierra agrícola en el APEC, con el objetivo de encontrar qué eco- 
nomía miembro puede producir al menos la misma cantidad de producción de alimentos con menos insumos de recursos, es decir, realizan un análisis de tipo input-oriented. Para ello utilizaron el análisis envolvente de datos. Los resultados muestran que la eficiencia general del uso de la tierra para la producción de alimentos es relativamente baja en la región del APEC, por lo que hay mucho espacio para que la mayoría de las economías aumenten la producción de alimentos con el nivel actual de insumos.

La misma temática de Yu et al. (2011) la analizan por Ayvar et al. (2018), quienes realizan un estudio de la eficiencia del sector agropecuario mexicano en el APEC, considerando la presencia de bad outputs durante el período 19802015. Para la medición de la eficiencia hicieron uso del análisis envolvente de datos, y para conocer la evolución de la productividad calcularon el índice Malmquist-Luenberger de Chung et al. (1997). Entre sus resultados se identifica que a lo largo del periodo de estudio el sector agropecuario de México fue más eficiente que el de otros países del APEC.

En el ámbito de las telecomunicaciones, un análisis común es el de brechas tecnológicas entre regiones y/o países. En este sentido es que Chun-Hsiung y Chun-Yu (2012) estudian las brechas tecnológicas, relacionadas con la eficiencia de los operadores de telecomunicaciones integrados del APEC, durante el periodo de 2001 a 2008, utilizando el enfoque de metafrontera del análisis envolvente de datos. Sus hallazgos reportan que los operadores del grupo de alta tasa de penetración tienen una mayor eficiencia técnica y brechas tecnológicas más pequeñas en promedio, que los del grupo de baja tasa de penetración.

La economía del conocimiento es una vertiente analítica de la ciencia económica, que busca colocar como centro de atención a la información, como elemento esencial para generar valor y riqueza, a través de transformarla en conocimiento. De ello, Ibne y Lawrey (2012) analizan eficiencia técnica y de escala de las economías basadas en el conocimiento, de la Asociación de Naciones del Sudeste Asiático (ASEAN, por sus siglas en inglés Association of South East Asian Nations) mediante la metodología de Data Envelopment Analysis para los años 1995 y 2010. Los resultados muestran que Filipinas y la República de Corea son los países más productivos en la utilización del conocimiento y $100 \%$ eficientes en uno o ambos años investigados.

Una de las características de la región del APEC es la importante cantidad de puertos con los que cuenta en la Cuenca del Pacífico, y que se relaciona con temas aduaneros y de movimiento de contenedores, por lo que es muy 
atractivo este objeto de estudio. En este sentido, Infante y Gutiérrez (2013) analizan los principales factores que determinan los niveles de eficiencia portuaria en las economías del APEC. Para ello emplean el análisis envolvente de datos para medir y evaluar la eficiencia de 33 puertos que pertenecen al APEC, en el periodo de 2003 a 2010. Entre sus hallazgos, ratifican que los resultados de eficiencia obtenidos dependen del tipo de modelo empleado que, a su vez, depende de una suposición sobre las propiedades de rendimiento a escala de la función de producción del puerto.

Así también Wu et al. (2016) analizan la productividad de los puertos de las economías del APEC. Para ello emplean la metodología del índice de productividad de Malmquist (1953) de metafrontera generalizada, para comparar la productividad portuaria de países desarrollados y países en desarrollo en el APEC. Los resultados indican que, en primer lugar, la tasa promedio de capacidad utilizada entre los puertos de los miembros del APEC fue solo del 65.7\% durante 2002-2011.

Por su parte, Zamora (2017) plantea identificar el grado de eficiencia o ineficiencia de las aduanas de 18 países de la región Asia Pacífico, incluyendo las aduanas de México, así como determinar el cambio tecnológico en las aduanas de la región. Para ello emplea la metodología del análisis envolvente de datos y el índice Malmquist (1953). Sus resultados muestran que los procesos de innovación y cambio tecnológico son fundamentales para la mejora de la productividad en las aduanas y que a pesar de ello no son el único factor que puede incidir en la mejora de eficiencia de éstas.

Otro análisis relacionado con los puertos es el que realizan Delfin y $\mathrm{Na}$ varro (2017), quienes miden la eficiencia de las terminales de contenedores de los principales puertos del APEC durante el periodo 2005-2013. Para ello emplean las propuestas metodológicas de Jondrow et al. (1982) y de Battese y Coelli (1988). Los resultados muestran que durante todo el periodo analizado, en promedio ningún puerto fue eficiente; sin embargo, Shanghái fue el que tuvo los resultados más altos, en contraste con el puerto de Callao en Perú, donde sus niveles de eficiencia fueron los más bajos.

La región del APEC también ha sido objeto de estudio en el ámbito del análisis de la eficiencia y la productividad, como lo muestra el trabajo de Ke (2017), donde se plantea evaluar la eficiencia de las economías miembros del APEC, durante 18 años, mediante la metodología propuesta por Tone y Tsutsui (2010), la cual sugiere un modelo de medida dinámica basada en holguras (sBM: Slacks-Based Measure). Entre sus resultados se identifica que 
durante el periodo de análisis su eficiencia promedio en 2008 es la mejor y que, en términos de áreas geográficas, las economías miembros de Oceanía tienen la mejor eficiencia.

El mismo objeto de estudio de Ke (2017) es analizado por Delfín y Navarro (2019), quienes dedican un capítulo de su obra al análisis de los métodos de estimación y cálculo de la productividad y el cambio tecnológico, con métodos paramétricos, considerando las estimaciones de frontera con métodos paramétricos, la estimación de frontera por medio de modelos determinísticos, las estimaciones de frontera por medio de modelos estocásticos y la medición de la productividad total de los factores con frontera estocástica; todos ellos para ser empleados en el análisis del APEC.

Finalmente, el análisis de la literatura sobre eficiencia y/o productividad para economías de la región Asia-Pacífico y/o del APEC lleva a identificar que también se ha incursionado en temas del medio ambiente, como se observa en el trabajo de Wang et al. (2016), quienes proponen medir la eficiencia económica y de las emisiones de $\mathrm{CO}_{2}$, de los miembros del APEC, mediante un modelo de evaluación de la eficiencia no radial. Sus resultados indican que la mayoría de los países tienen una eficiencia económica extremadamente alta, pero una eficiencia de emisiones de $\mathrm{CO}_{2}$ relativamente baja y una eficiencia integrada, excepto en Estados Unidos, Japón y Singapur.

Derivado de lo anterior, esta revisión de literatura permite observar que es relativamente amplio el análisis de la eficiencia y la productividad en la Cuenca del Pacífico y en el marco del APEC; no obstante, no se identifican investigaciones sobre el efecto que tiene el movimiento de TEU sobre la eficiencia y la productividad total de los factores, dejando un hueco analítico para el entendimiento de la región del APEC o para la propia Cuenca del Pacífico, por lo que en este documento se emplea la metodología de Análisis de Fronteras Estocásticas para analizar este fenómeno.

\section{Metodología}

La ciencia económica aporta el concepto de frontera de producción, la cual es determinada por la función de producción, representando el máximo producto que se puede obtener con un conjunto de insumos. Una ventaja que proporciona estimar la frontera de producción consiste en que se puede observar si se está operando eficientemente o, dada su tecnología de producción, existe la posibilidad de mejorar. Uno de los estudios primigenios en relación con el 
análisis de las fronteras de producción se encuentra en Farrell (1957), quien aporta una definición de eficiencia de la empresa, argumentando que uno de los componentes es la eficiencia técnica, siendo ésta la que refleja la habilidad para obtener la máxima producción, dados sus insumos productivos.

Un acercamiento paramétrico para la medición de la eficiencia es proporcionado por Aigner et al. (1977), el cual dio como resultado el desarrollo del modelo de fronteras estocásticas. Es así que para el desarrollo de esta investigación se emplea una función de producción de frontera estocástica para la obtención de la eficiencia técnica, ya que el interés radica en estimarla y conocer si es posible aumentar la producción sin alterar la cantidad de insumos en el proceso.

Particularmente se hace uso de la metodología propuesta por Battese y Coelli (1995), quienes emplean un modelo para los efectos de la ineficiencia técnica, en una función de producción de frontera estocástica, para un panel de datos. Battese y Coelli (1995) plantean una función de producción de frontera estocástica, como la que se presenta en la ecuación 1.

$Y_{i t}=\exp \left(x_{i t} \beta+V_{i t}-U_{i t}\right) ; i=1, \ldots, N ; t=1, \ldots, T$

Que en el contexto de este manuscrito $Y_{i t}$ se refiere a la producción para la i-ésima economía del APEC considerada, de la t-ésima observación a través del tiempo, $x_{i t}$ es una matriz de valores de una función de insumos -inversión y empleo-y otras variables explicativas asociadas con la i-ésima economía en el tiempo t. $\beta$ es un vector de parámetros a ser estimados, $V_{i t}$ son los errores aleatorios que se suponen independientes e idénticamente distribuidos, $\mathrm{y}$ que se distribuyen como $N\left(0, \sigma_{v}^{2}\right)$ e independientemente distribuidos de $U_{i t}$, siendo $U_{i t}$ un conjunto de variables aleatorias no negativas, asociadas con la ineficiencia técnica de la producción, las cuales se suponen independientemente distribuidas.

La ecuación 1 representa la función de producción de frontera estocástica. En tanto que los efectos de la ineficiencia técnica, los $U_{i t}$ 's, se considera que son representados por un grupo de variables explicativas, las $z_{i t}{ }^{\prime}$ 's y un vector de coeficientes desconocidos, $\delta$. Así, la ecuación de ineficiencia técnica, $U_{i t}$ en el modelo de frontera estocástica 1, siguiendo a Battese y Coelli (1995), se especifica en la ecuación 2.

$U_{i t}=z_{i t}+W_{i t}$ 
Donde $z_{i t}$ es un vector de variables explicativas asociadas con la ineficiencia técnica de la producción de las economías a través del tiempo — una variable de tendencia lineal, un conjunto de variables dummy para rescatar la posible heterogeneidad existente entre las diferentes economías del APEC objeto de estudio, y una variable más, que registra información sobre el movimiento de contenedores en la región-, y $\delta$ es un vector de parámetros a estimarse y $W_{i t}$ una variable aleatoria.

Para la estimación simultánea de los parámetros de la frontera estocástica y del modelo de efectos de la ineficiencia técnica, Battese y Coelli (1995) proponen el método de máxima verosimilitud. ${ }^{5}$ De ello, la función de verosimilitud se expresa en función de la varianza de los parámetros.

$\sigma_{s}^{2}=\sigma^{2}{ }_{v}+\sigma^{2} ; \gamma=\frac{\sigma^{2}}{\sigma_{S}^{2}}$

Por tanto, la eficiencia técnica de la producción, para la i-ésima economía en el momento t se expresa por la ecuación siguiente:

$E T_{i t}=\exp \left(-U_{i t}\right)=\exp \left(-z_{i t}-W_{i t}\right)$

Asimismo, la medida de eficiencia técnica, relativa a la función de producción 1) para un panel de datos se define en la ecuación 3 , siguiendo a Coelli (1996):

$E T_{i t}=\frac{E\left(Y_{t}^{*} / u_{t}, X_{t}\right)}{E\left(Y_{t}^{*} / u_{t}=0, X_{t}\right)}=\exp \left(-u_{t}\right)$

Donde $Y_{i t}^{*}$ es la producción de la i-ésima economía. De ello, la eficiencia técnica se calcula como el cociente entre la producción obtenida y la máxima obtenible, dadas las cantidades de los insumos (es decir, cuando $u_{i t}=0$ ). Su valor oscila entre 0 y 1 , siendo este último caso cuando se es totalmente eficiente.

\section{Datos y fuentes de información}

Los datos de producción, inversión y empleo proceden de la base de datos del Banco Mundial (World Bank, 2020), que se corresponden con el producto interno bruto, en dólares a precios constantes de 2010, la formación bruta

5. La función de verosimilitud y las derivadas parciales respecto a los parámetros del modelo son desarrollados en Battese y Coelli (1993).

154 México y la Cuenca del Pacífico. Vol 10, núm. 30 / septiembre-diciembre de 2021. 
de capital fijo, en dólares a precios corrientes y la fuerza laboral total, respectivamente. Los datos del número de contenedores, en TEU, proceden de la base de datos de la UNCTAD (2020a).

Para el análisis se ha considerado a las economías del APEC que se encuentran entre las 30 principales operadoras de TEUs del mundo, las cuales son presentadas en la tabla 3 , donde también se muestra la posición que ocupan en el ámbito mundial.

Tabla 3

Principales 30 operadores de TEUs en la región del APEC

\begin{tabular}{c|l|c|l}
\hline Posición & \multicolumn{1}{|c|}{ País } & Posición & \multicolumn{1}{c}{ País } \\
\hline 1 & China & 13 & China, Taiwán Provincia de \\
\hline 2 & Estados Unidos de América & 15 & Indonesia \\
\hline 3 & Singapur & 18 & Tailandia \\
\hline 4 & Corea, República de & 22 & Australia \\
\hline 5 & Malasia & 24 & Filipinas \\
\hline 6 & Japón & 26 & México \\
\hline 7 & China, Hong Kong SAR & 28 & Canadá \\
\hline 12 & Vietnam & 30 & Federación Rusa \\
\hline
\end{tabular}

Fuente: elaboración de los autores con datos de la UNCTAD (2020a).

\section{Resultados}

Para determinar el efecto que tiene el número de contenedores que desplaza cada economía del APEC, sobre su eficiencia técnica, la primera etapa en su estimación consiste en seleccionar la mejor tecnología de producción para la función de producción de frontera estocástica. Para ello se realiza un contraste de especificación para elegir entre la disyuntiva de una función trascendental logarítmica o una Cobb-Douglas, y otros más para seleccionar las variables a incluir en la ecuación de ineficiencia.

Dichos contrastes se realizan a partir de la función de verosimilitud de cada modelo, para obtener el estadístico $\lambda=-2\left[\log \left(f\right.\right.$.verosimilitud $\left.\left(\mathrm{H}_{\mathrm{o}}\right)\right)-\log (\mathrm{f}$. verosimilitud $\left.\left.\left(\mathrm{H}_{1}\right)\right)\right]$. Así también, se estiman dos modelos: uno sin restricciones y otro restringido.

En la tabla 4 se presenta el conjunto de contrastes que permiten elegir la mejor tecnología de producción, y las variables a incluir en la ecuación de ineficiencia. De ello, el primero se realiza para elegir la tecnología de produc- 
ción más adecuada, considerando en la ecuación de ineficiencia una variable de tendencia, así como un conjunto de variables que permiten identificar la existencia de heterogeneidades entre las economías analizadas. También se incluye la variable que recoge información sobre el número de contenedores que desplaza anualmente cada economía.

El resultado del primer contraste permite la decisión de rechazar la hipótesis nula de que la tecnología de producción más adecuada es la Cobb Douglas. Asimismo, en el modelo restringido no se considera la variable que recoge información sobre el número de contenedores que desplaza anualmente cada economía, y la decisión es también que la tecnología de producción adecuada es una función trans-log, al rechazar la hipótesis nula de que los cuadrados de las variables explicativas y los productos cruzados son iguales a cero.

Una vez elegida la forma funcional adecuada, se realiza el segundo contraste para determinar qué variables son relevantes para incluir en la ecuación de ineficiencia. Para ello se incluye el intercepto, una variable de tendencia, así como ficticias dicotómicas (o dummies) para modelar la posible heterogeneidad existente entre las economías en análisis. También se incluye la variable que recoge información sobre el número de contenedores que desplaza cada economía considerada en el estudio, siendo ésta la que, al no incluirse en la ecuación de ineficiencia, genera al modelo restringido.

El segundo contraste muestra que en el caso del modelo no restringido se rechaza la hipótesis nula de que todos los parámetros de la ecuación de ineficiencia son simultáneamente iguales a cero, por lo que esto justifica su inclusión. En el caso del modelo restringido, también se corrobora la importancia de incluir tanto al intercepto como a la variable de tendencia y las dummies.

El tercer contraste valida la inclusión de la variable de tendencia y las dummies, tanto en el modelo no restringido como en el restringido, ya que el resultado permite rechazar la hipótesis nula de que éste es no significativo al 95\% de confianza. El cuarto contraste analiza la relevancia de incluir en el modelo no restringido, simultáneamente a las variables dummy individuales que recogen la posible heterogeneidad entre economías, así como a la variable que recoge información sobre el número de contenedores que desplaza cada una de ellas. El resultado de éste, que rechaza la hipótesis nula, es que es adecuado incluirlas en la estimación.

El cuarto contraste para el modelo restringido analiza la relevancia de incluir las variables dummy en el modelo. El resultado indica que al rechazar 
la hipótesis nula, es correcto incluirlas como caracterizadoras de la heterogeneidad entre las economías analizadas en el estudio.

\section{Tabla 4}

Contrastes de especificación, modelos restringido y no restringido.

Restricción: número de TEUs $\left(\delta_{16}\right)$

\begin{tabular}{|c|c|c|c|c|c|c|c|}
\hline \multirow[t]{2}{*}{ Hipótesis nula } & \multicolumn{2}{|c|}{ Log. F. verosimilitud } & \multicolumn{2}{|c|}{ Valor $\lambda$} & \multicolumn{2}{|c|}{ Valor crítico } & Decisión \\
\hline &  &  & 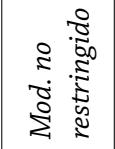 &  & 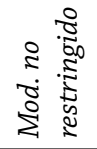 & 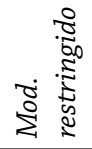 & $95 \%$ \\
\hline $\begin{array}{l}H_{0}: \beta_{K, L}=\beta_{L}^{2}=\beta_{K}^{2}=0 \\
H_{0}: \beta_{K, L}=\beta_{L}^{2}=\beta_{K}^{2}=0\end{array}$ & 162.23 & 384.86 & 288.45 & 161.74 & 7.81 & 7.81 & $\begin{array}{l}\text { Rechazo/ } \\
\text { Rechazo }\end{array}$ \\
\hline $\begin{array}{l}H_{0}: \gamma=\delta_{0}=\ldots=\delta_{16}=0 \\
H_{0}: \gamma=\delta_{0}=\ldots=\delta_{15}=0\end{array}$ & 50.10 & -145.32 & 512.72 & 1222.10 & 28.86 & 32.07 & $\begin{array}{l}\text { Rechazo/ } \\
\text { Rechazo }\end{array}$ \\
\hline $\begin{array}{l}H_{0}: \delta_{1}=\ldots=\delta_{16}=0 \\
H_{0}: \delta_{1}=\ldots=\delta_{15}=0\end{array}$ & 290.17 & -145.32 & 32.58 & 71.88 & 26.29 & 30.14 & $\begin{array}{l}\text { Rechazo/ } \\
\text { Rechazo }\end{array}$ \\
\hline $\begin{array}{l}H_{0}: \delta_{2}=\ldots=\delta_{16}=0 \\
H_{0}: \delta_{2}=\ldots=\delta_{15}=0\end{array}$ & 50.33 & -99.61 & 512.25 & 69.93 & 27.58 & 28.86 & $\begin{array}{l}\text { Rechazo/ } \\
\text { Rechazo }\end{array}$ \\
\hline
\end{tabular}

Nota: el estadístico $\lambda$ se calcula como: $\lambda=-2\left[\log \left(f\right.\right.$.verosimilitud $\left.\left(H_{0}\right)\right)-\log \left(f\right.$.verosimilitud $\left.\left(H_{1}\right)\right)$, el cual tiene una distribución una chi-cuadrada, con grados de libertad correspondientes al número de parámetros que se igualan a cero en la hipótesis nula. En el contraste cuya hipótesis nula considera $\gamma=0$ el estadístico $\lambda$ sigue una distribución chi-cuadrada mixta. Fuente: elaboración de los autores con datos del Kodde \& Palm (1986), World Bank (2020) y UNCTAD (2020a).

Una vez elegida la mejor tecnología de producción, y las variables a incluir en la ecuación de ineficiencia, y siguiendo la propuesta metodológica de Battese y Coelli (1995) y Greene (1993), se estima la eficiencia técnica de las economías del APEC que cuentan con puertos para el manejo de TEUs, que se encuentran entre los 30 principales en el mundo.

Los resultados indican que considerar el movimiento de TEUs en la ecuación de ineficiencia contribuye, en general, a mejorar la eficiencia de las economías, como se puede observar en la figura 2, donde se compara la eficiencia media de éstos a través del tiempo. En un primer momento se estima 
la eficiencia sin considerar el movimiento de TEUs, y en un segundo momento teniéndolo en consideración para cada economía. El nivel de eficiencia mejora en poco más de 1.5 puntos si sucede esto último.

\section{Figura 2}

Evolución temporal de la eficiencia técnica de las principales economías del APEC con puertos para contenedores

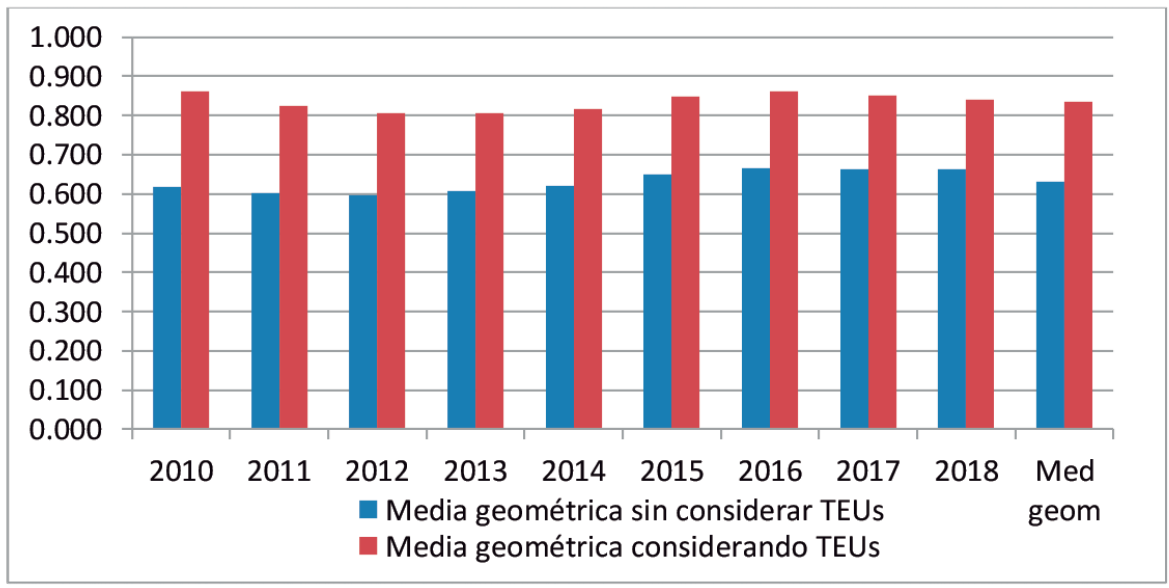

Fuente: elaboración de los autores con datos de World Bank (2020) y UNCTAD (2020a).

Para identificar si los niveles de eficiencia técnica tienden a converger a través del tiempo entre economías, se calcula su desviación estándar. Si ésta aumenta, ello indica la existencia de un proceso de divergencia en el uso de los factores de la producción; y si, por el contrario, ésta tiende a disminuir, será indicativo de un proceso de convergencia. Así, la evolución temporal de la desviación estándar se presenta en la figura 3. Como se puede observar, es apenas notoria la tendencia positiva en ambas líneas, lo que indica un proceso marginal de divergencia. Asimismo, al observar los coeficientes pendientes en las ecuaciones de la recta de regresión se puede observar que la correspondiente a la recta con TEUs es marginalmente mayor, lo que indica que si bien incluir a los TEUs en la función de producción de frontera estocástica contribuye a mejorar la eficiencia, también genera un marginal proceso de divergencia en eficiencia entre las economías del APEC. 


\section{Figura 3}

Desviación estándar de la evolución temporal de la eficiencia técnica de las principales economías del APEC con puertos para contenedores

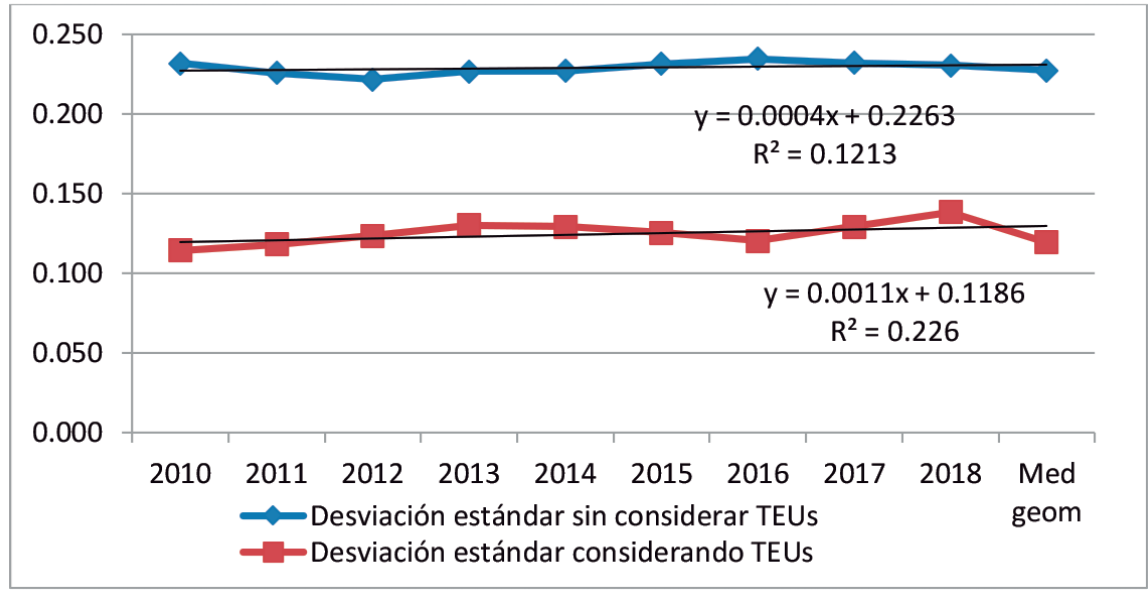

Fuente: elaboración de los autores con datos de World Bank (2020) y UNCTAD (2020a).

Entre economías, el efecto de considerar en la función de producción el número de TEUs que desplazan las economías del APEC, considerados en este estudio, es heterogéneo, como se puede observar en el figura 4. Economías como la de China, la de Indonesia, la de México, la de Filipinas, la de Rusia y la de Vietnam se ven fuertemente favorecidas en su nivel de eficiencia técnica, en tanto otras, como la de Australia, la de Canadá, la de Hong Kong, la de Singapur y la de Estados Unidos se ven con pérdidas en el uso de los factores de la producción, reduciendo su nivel de eficiencia técnica. 


\section{Figura 4}

Eficiencia técnica de las principales economías del APEC con puertos para contenedores

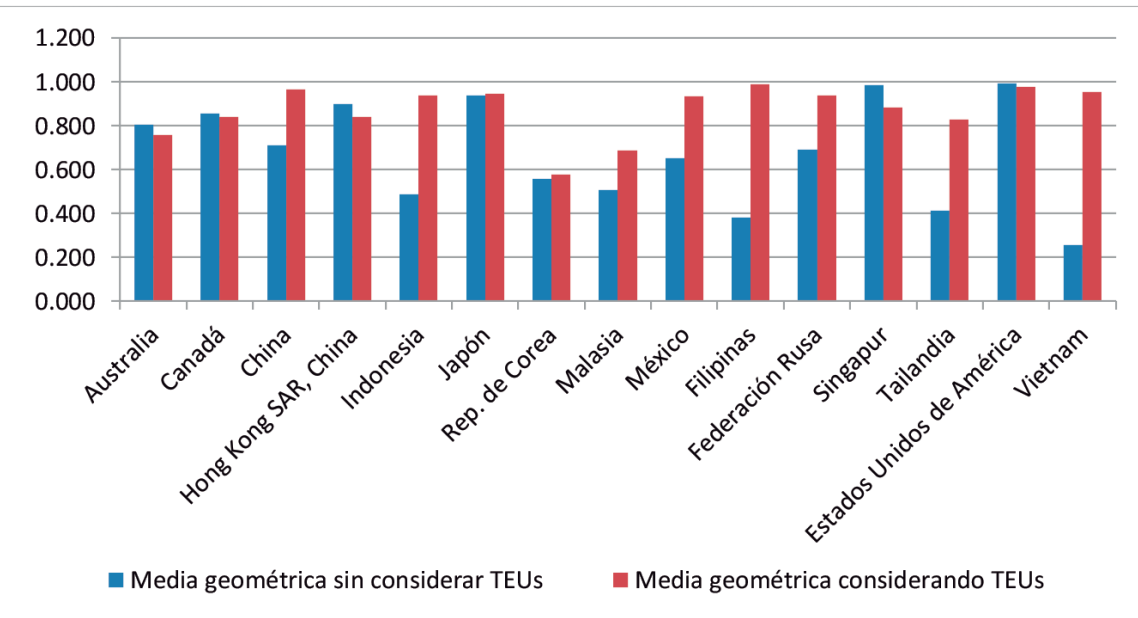

Fuente: elaboración de los autores con datos de World Bank (2020) y UNCTAD (2020a).

La eficiencia técnica individual de las economías del APEC se presenta en la tabla 5. Ésta se construye sin considerar el efecto que tiene la variable de número de contenedores que desplaza cada economía, cada año. Los resultados muestran que al final del periodo de análisis las economías de Estados Unidos y Singapur alcanzan niveles altos, del 99 y $98 \%$ de eficiencia, en tanto que las menos eficientes son la de Vietnam y Filipinas, con 25 y 38 puntos porcentuales, respectivamente. La economía de México alcanza un nivel de eficiencia de 65 puntos porcentuales en el año 2018. 


\section{Tabla 5}

Indicador de eficiencia técnica de las principales economías del APEC con puertos de contenedores. Sin considerar TEUs en la ecuación de ineficiencia

\begin{tabular}{|c|c|c|c|c|c|c|c|c|c|c|}
\hline & 2010 & 2011 & 2012 & 2013 & 2014 & 2015 & 2016 & 2017 & 2018 & $\begin{array}{c}\text { Media } \\
\text { geométrica }\end{array}$ \\
\hline Australia & 0.792 & 0.754 & 0.725 & 0.727 & 0.777 & 0.826 & 0.897 & 0.896 & 0.879 & 0.806 \\
\hline Canadá & 0.812 & 0.794 & 0.785 & 0.801 & 0.825 & 0.899 & 0.931 & 0.924 & 0.926 & 0.853 \\
\hline China & 0.765 & 0.713 & 0.693 & 0.677 & 0.679 & 0.707 & 0.740 & 0.728 & 0.694 & 0.710 \\
\hline $\begin{array}{l}\text { Hong Kong } \\
\text { SAR, China }\end{array}$ & 0.910 & 0.891 & 0.861 & 0.878 & 0.888 & 0.900 & 0.917 & 0.922 & 0.927 & 0.899 \\
\hline Indonesia & 0.459 & 0.442 & 0.447 & 0.472 & 0.492 & 0.517 & 0.519 & 0.522 & 0.535 & 0.488 \\
\hline Japón & 0.906 & 0.864 & 0.864 & 0.943 & 0.958 & 0.980 & 0.970 & 0.975 & 0.973 & 0.936 \\
\hline $\begin{array}{l}\text { Corea, } \\
\text { República }\end{array}$ & 0.558 & 0.551 & 0.561 & 0.561 & 0.554 & 0.569 & 0.570 & 0.548 & 0.555 & 0.559 \\
\hline Malasia & 0.498 & 0.487 & 0.469 & 0.469 & 0.482 & 0.521 & 0.539 & 0.552 & 0.553 & 0.507 \\
\hline México & 0.626 & 0.605 & 0.610 & 0.623 & 0.632 & 0.663 & 0.699 & 0.701 & 0.699 & 0.650 \\
\hline Filipinas & 0.372 & 0.377 & 0.372 & 0.373 & 0.382 & 0.385 & 0.381 & 0.391 & 0.391 & 0.380 \\
\hline $\begin{array}{l}\text { Federación } \\
\text { Rusa }\end{array}$ & 0.678 & 0.613 & 0.606 & 0.601 & 0.648 & 0.797 & 0.806 & 0.743 & 0.766 & 0.691 \\
\hline Singapur & 0.984 & 0.982 & 0.980 & 0.979 & 0.980 & 0.985 & 0.987 & 0.988 & 0.990 & 0.984 \\
\hline Tailandia & 0.402 & 0.382 & 0.384 & 0.395 & 0.410 & 0.424 & 0.437 & 0.438 & 0.436 & 0.411 \\
\hline $\begin{array}{l}\text { Estados } \\
\text { Unidos }\end{array}$ & 0.994 & 0.993 & 0.992 & 0.992 & 0.991 & 0.991 & 0.992 & 0.991 & 0.991 & 0.992 \\
\hline Vietnam & 0.229 & 0.243 & 0.250 & 0.253 & 0.255 & 0.261 & 0.270 & 0.274 & 0.278 & 0.257 \\
\hline $\begin{array}{l}\text { Media } \\
\text { geométrica }\end{array}$ & 0.618 & 0.602 & 0.598 & 0.606 & 0.620 & 0.650 & 0.665 & 0.662 & 0.663 & 0.631 \\
\hline
\end{tabular}

Nota: al multiplicar por 100 el indicador de eficiencia se puede obtener una lectura en términos porcentuales.

Fuente: elaboración de los autores con datos de World Bank (2020) y UNCTAD (2020a).

Cuando se considera el efecto del número de contenedores que desplaza cada economía cada año, en la eficiencia de éstas, se observa que ésta contribuye a su mejoría, sobre todo en las economías menos desarrolladas de la región del APEC. Esto se puede observar en la tabla 6, la cual muestra la eficiencia técnica a través del tiempo. Economías como la de México, Filipinas, Tailandia y Vietnam ven mejorada su eficiencia de manera significativa, en tanto que en economías como la de Japón y la República de Corea apenas es perceptible. Sin embargo, otras como las de Australia, Canadá, Hong Kong y Estados Unidos pierden eficiencia. 


\section{Tabla 6}

Indicador de eficiencia técnica de las principales economías del APEC con puertos de contenedores. Considerando TEUs en la ecuación de ineficiencia

\begin{tabular}{|c|c|c|c|c|c|c|c|c|c|c|}
\hline & 2010 & 2011 & 2012 & 2013 & 2014 & 2015 & 2016 & 2017 & 2018 & $\begin{array}{c}\text { Media } \\
\text { geométrica }\end{array}$ \\
\hline Australia & 0.758 & 0.718 & 0.685 & 0.686 & 0.731 & 0.777 & 0.846 & 0.842 & 0.821 & 0.760 \\
\hline Canadá & 0.839 & 0.814 & 0.800 & 0.814 & 0.837 & 0.908 & 0.932 & 0.922 & 0.921 & 0.864 \\
\hline China & 0.966 & 0.888 & 0.815 & 0.748 & 0.707 & 0.711 & 0.731 & 0.662 & 0.593 & 0.750 \\
\hline $\begin{array}{l}\text { Hong Kong } \\
\text { SAR, China }\end{array}$ & 0.838 & 0.804 & 0.772 & 0.790 & 0.796 & 0.811 & 0.828 & 0.823 & 0.825 & 0.810 \\
\hline Indonesia & 0.938 & 0.881 & 0.872 & 0.915 & 0.939 & 0.958 & 0.952 & 0.948 & 0.955 & 0.928 \\
\hline Japón & 0.945 & 0.917 & 0.911 & 0.956 & 0.963 & 0.975 & 0.968 & 0.970 & 0.967 & 0.952 \\
\hline $\begin{array}{l}\text { Corea, } \\
\text { República }\end{array}$ & 0.578 & 0.560 & 0.568 & 0.562 & 0.550 & 0.563 & 0.559 & 0.529 & 0.530 & 0.555 \\
\hline Malasia & 0.688 & 0.652 & 0.607 & 0.601 & 0.614 & 0.678 & 0.705 & 0.718 & 0.705 & 0.662 \\
\hline México & 0.935 & 0.896 & 0.897 & 0.912 & 0.918 & 0.945 & 0.965 & 0.963 & 0.959 & 0.932 \\
\hline Filipinas & 0.990 & 0.990 & 0.988 & 0.987 & 0.987 & 0.986 & 0.983 & 0.983 & 0.981 & 0.986 \\
\hline $\begin{array}{l}\text { Federación } \\
\text { Rusa }\end{array}$ & 0.939 & 0.824 & 0.797 & 0.776 & 0.856 & 0.979 & 0.979 & 0.957 & 0.963 & 0.893 \\
\hline Singapur & 0.881 & 0.861 & 0.841 & 0.835 & 0.833 & 0.869 & 0.882 & 0.892 & 0.904 & 0.866 \\
\hline Tailandia & 0.828 & 0.751 & 0.727 & 0.740 & 0.777 & 0.806 & 0.826 & 0.805 & 0.780 & 0.781 \\
\hline $\begin{array}{l}\text { Estados } \\
\text { Unidos }\end{array}$ & 0.976 & 0.973 & 0.967 & 0.963 & 0.953 & 0.950 & 0.950 & 0.942 & 0.929 & 0.956 \\
\hline Vietnam & 0.951 & 0.970 & 0.971 & 0.969 & 0.963 & 0.960 & 0.965 & 0.957 & 0.948 & 0.962 \\
\hline $\begin{array}{l}\text { Media } \\
\text { geométrica }\end{array}$ & 0.861 & 0.824 & 0.805 & 0.806 & 0.817 & 0.848 & 0.862 & 0.850 & 0.839 & 0.834 \\
\hline
\end{tabular}

Fuente: elaboración de los autores con datos de World Bank (2020) y UNCTAD (2020a).

Derivado de lo anterior, los resultados indican que en general aún existe la posibilidad de mejorar en el uso de los factores de la producción, dado que el nivel de eficiencia de las economías no logra llegar en términos porcentuales al 100\%, no obstante que ésta mejora en la mayoría de ellas al considerar el efecto del movimiento de TEUs en sus puertos. 


\section{Conclusiones}

La importancia económica de la Cuenca del Pacífico radica en su dinamismo. En ésta, el APEC, integrado por 21 economías, cuenta con más de 3,000 millones de personas, con alrededor del $60 \%$ del producto interno bruto mundial y el $50 \%$ del comercio internacional. Muchos de los principales exportadores e importadores en contenedores son miembros de este organismo de cooperación. También en esta región se localizan las principales rutas marítimas comerciales en Asia-Pacífico. Basta decir que de los 50 principales puertos con movimiento de contenedores del mundo, 35 se encuentran en economías del APEC.

Asimismo, por su importancia esta región ha inspirado múltiples estudios en diversas temáticas, entre ellas, sin ser limitativas, las relacionadas con el sector externo, la prospectiva, la economía regional, o bien la salud mental o el medio ambiente. Otros temas objeto de estudio se han relacionado con el ahorro energético, el transporte, o el sector agropecuario. Algunos más relacionados con la eficiencia y productividad. La revisión del estado del objeto de estudio ha permitido identificar diversos estudios sobre estos temas; sin embargo, no se ha hallado alguno que permita observar el efecto que tiene el movimiento de contenedores en la eficiencia técnica de los países, por lo que este análisis ha sido abordado en este documento con el objeto de contribuir analíticamente al entendimiento, desde esta óptica, de la región de la Cuenca del Pacífico y, de manera particular, a la región del APEC.

Derivado de lo anterior, se ha estimado la eficiencia técnica de las economías del APEC que se encuentran entre los 30 principales operadores de TEUs de la región. Para ello se ha empleado la metodología de Análisis de Fronteras Estocásticas, encontrando que a través del tiempo la eficiencia técnica media de los países ha aumentado y que ésta también mejora cuando se considera el efecto que tiene la capacidad de movimiento de contenedores de cada economía. Sin embargo, al analizar la posibilidad de que exista un proceso de convergencia en eficiencia, se identifica que, por el contrario, existe un marginal proceso de divergencia en la eficiencia de las economías analizadas, que cuentan con puertos para contenedores. Este proceso de divergencia permite inferir que existe una significativa heterogeneidad entre las economías analizadas que también se ve expresada en el movimiento de contenedores que cada una de ellas realiza. 
El análisis del efecto de los puertos de contenedores en la región del APEC, y el número de TEUs que desplaza anualmente cada economía, sobre su eficiencia, permite argumentar que éste es favorable para la mayoría de ellas; sin embargo, las economías menos favorecidas son las de mayores rentas per cápita. De lo anterior se puede concluir que el dinamismo en el movimiento de TEUs tiene un efecto favorecedor en la reducción de las ineficiencias de las economías del APEC y, en mayor medida, para las economías de menor renta per cápita.

\section{Referencias bibliográficas}

Aigner, D. J., Lovell, C. A. K., \& Schmidt, P. (1977). Formulation and estimation of stochastic frontier production function models. Journal of Econometrics, 6(1): 21-37. https://doi.org/10.1016/0304-4076(77)90052-5

Anees, H. Z. S., Wasif, Z. M., Shahbaz, M., \& Hou, F. (2019). Dynamic linkages between globalization, financial development and carbon emissions: Evidence from Asia Pacific Economic Cooperation countries. Journal of Cleaner Production, 228, 533-543. https://doi.org/10.1016/j. jclepro.2019.04.210

Ansari, T. U., Wild, O., Li J., Yang, T., Xu, W., Sun, Y., \& Wang, Z. (2019). Effectiveness of short-term air quality emission controls: a high-resolution model study of Beijing during the Asia-Pacific Economic Cooperation (APEC) summit. Atmospheric Chemistry and Physics, 19(13), 8651-8668. https://doi.org/10.5194/acp-19-8651-2019

APEC Secretariat. (2008). Survey of Policies and Programs that Promote Fuel-Efficient Transport in APEC Economies. The Alliance to Save Energy. https://www. APEC.org/Publications/2008/09/Survey-of-Policies-and-Programs-thatPromote-FuelEfficient-Transport-in-APEC-Economies-May-2008-Publ

Ayvar, C. F. J., Navarro, C. J. C. L., \& Zamora, T. A. I. (2018). El Sector Agropecuario Mexicano en APEC: Un análisis a través de la envolvente de datos con presencia de bad outputs. Análisis Económico, 33(83), 125-143. https:// doi.org/10.24275/uam/azc/dcsh/ae/2018v33n83/ayvar

Battese, G. E. \& Coelli, T. J. (1988). Prediction of Firm Level Technical Efficiencies with a Generalized Frontier Production Function and Panel Data. Journal of Econometrics, 38(3), 387-399. https://doi.org/10.1016/03044076(88)90053-X 
Battese, G. E. \& Coelli, T. J. (1993). A Stochastic Frontier Production Function incorporating a model for technical inefficiency effects (Working Paper in Econometrics and Applied Statistics 69/93). Department of Econometrics, University of New England.

Battese, G. E. \& Coelli, T. J. (1995). A model for technical inefficiency effects in a stochastic frontier production function for panel data. Empirical Economics, 20, 325-332. https://doi.org/10.1007/BF01205442

Chaekwang, Y. (2019). Assessing APEC in an Era of US-China Rivalry -APEC's Transition from a Talking Shop to a Showroom-. Journal of APEC Studies, 11(2), 1-14. http://scholar.dkyobobook.co.kr/searchDetail. laf?barcode $=4010027500894$

Chiang, R. P.Y., Lin, C. F., Li, Y. C., \& Hsu, M. H. (2019). The importance of sleep for governmental sectors, general population and industry - Asia-Pacific Economic Cooperation (APEC) Sleep Technology Agenda. Sleep Medicine Reviews, 43, 135-136. https://doi.org/10.1016/j.smrv.2018.10.002

Chung, Y. H., Färe, R., \& Grosskopf, S. (1997). Productivity and Undesirable Outputs: A Directional Distance Function Approach. Journal of Environmental Management, 51(3), 229-240. https://doi.org/10.1006/ jema.1997.0146

Chun-Hsiung, L. \& Chun-Yu, L. (2012). Measuring the technology gap of APEC integrated telecommunications operators. Telecommunications Policy, 36(10-11), 989-996. https://doi.org/10.1016/j.telpol.2012.05.002

Coelli, T. (1996). A Guide to FRONTIER Version 4.1: A Computer Program for Stochastic Frontier Production and Cost Function Estimation (CEPA Working Paper 96/07). Center for Efficiency and Productivity Analysis. http://tarjomefa.com/wp-content/uploads/2017/07/7209-English-TarjomeFa.pdf

Delfin, O. O. V. \& Navarro, C. J. C. L. (2017). La eficiencia de los puertos en la región del APEC, 2005-2013: una medición a través del Análisis de la Frontera Estocástica (SFA). México y la Cuenca del Pacífico, 6(16), 19-50. https://doi.org/10.32870/mycp.v6i16.519

Delfín, O. O. V., \& Navarro, C. J. C. L. (2019). Productividad y eficiencia en los puertos de la región del APEC. Universidad Michoacana de San Nicolás de Hidalgo; Juan Pablos Editor.

Dowrick, C., Kassai R. K., Lam C. L. K., Lam, R. W., Manning, G., Murphy, J., Ng C. H., \& Thuraisingham, C. (2020). The APEC Digital Hub-WONCA Collaborative Framework on Integration of Mental Health into Primary 
Care in the Asia Pacific. Journal of Multidisciplinary Healthcare, 2020(13), 1693-1704. https://doi.org/10.2147/JMDH.S271070

Erkekoğlu, H., \& Yilmaz, B. (2019). Investigation of Foreign Trade between Turkey and Member Countries of Asia-Pacific Economic Cooperation (APEC) with Gravity Model. Journal of Yaşar University, 14(54), 141-151. https://doi.org/10.19168/jyasar.525789

Farrell, M. J. (1957). The Measurement of Productive Efficiency. Journal of the Royal Statistical Society, 120(3), 253-290. https://doi. org $/ 10.2307 / 2343100$

Greene, W. H. (1993). The Econometric Approach to Efficiency Analysis. En H. Fried, K. Lovell, \& S. Schmidt (Eds.), The Measurement of Productive Efficiency (pp. 92-250). Oxford University Press. 10.1093/acprof:oso/9780195183528.001.0001

Huang, C.J., \& Liu, J.T. (1994). Estimation of a non-neutral stochastic frontier production function. Journal of Productivity Analysis, 5, 171-180. https:// doi.org/10.1007/BF01073853

Ibne, A. M. N., \& Lawrey, R. (2012). Evaluating the Comparative Performance of Technical and Scale Efficiencies in Knowledge-Based Economies (KBEs) in ASEAN: A Data Envelopment Analysis (DEA) Application. European Journal of Economics, Finance and Administrative Sciences, (51), 82-96. https:// www.europeanjournalofeconomicsfinanceandadministrativesciences. com/issues/EJEFAS_51.html

Infante, Z., \& Gutiérrez, A. (2013). Port Efficiency in APEC. México y la Cuenca del Pacífico, 2(3), 41-73. https://doi.org/10.32870/mycp.v2i3.397

Jin-Li, H., \& Chih-Hung, K. (2007). Efficient energy-saving targets for APEC economies. Energy Policy, 35(1), 373-382. https://doi.org/10.1016/j. enpol.2005.11.032

Jondrow, J., Lovell, K., Materov, I., \& Schmidt, P. (1982). On the estimation of technical inefficiency in the stochastic frontier production function model. Journal of Econometrics, 19(2-3), 233-238. https://doi. org/10.1016/0304-4076(82)90004-5

Ke, T. (2017). Energy efficiency of APEC members - applied dynamic SBM model. Carbon Management, 8(4), 293-303. https://doi.org/10.1080/17 583004.2017.1357401

Kodde, D. A., \& Palm, F. C. (1986). Wald Criteria for Jointly Testing Equality and Inequality Restrictions. Econometrica, 54(5), 1243-1248. https://doi. org/10.2307/1912331 
Malmquist, S. (1953). Index numbers and indifference surfaces. Trabajos de Estadística, 4(2), 209-242. https://doi.org/10.1007/BF03006863

Sun, Y., Zhu, L., Xu, Z., Xiao, L., Zhang, J., \& Zhang, J. (2019). Characteristic analysis and forecast of electricity supply and demand in APEC. Global Energy Interconnection, 2(5), 413-422. https://doi.org/10.1016/j. gloei.2019.11.016

Tone, K., \& Tsutsui, M. (2010). Dynamic DEA: A Slacks-Based Measure approach. Omega, 38(3-4), 145-156. https://doi.org/10.1016/j.omega.2009.07.003

United Nations Conference on Trade and Development (UNCTAD). (2018). Informe Sobre el Transporte Marítimo 2018. Naciones Unidas. https:// UNCTAD.org/system/files/official-document/rmt2018_es.pdf

United Nations Conference on Trade and Development (UNCTAD). (2019). Informe Sobre el Transporte Marítimo 2019. Naciones Unidas. https:// UNCTAD.org/system/files/official-document/rmt2019_es.pdf

United Nations Conference on Trade and Development (UNCTAD). (2020a). Container Port Throughput, Annual [Database]. United Nations. http://unctadstat.UNCTAD.org/wds/TableViewer/tableView. aspx?ReportId $=13321$

United Nations Conference on Trade and Development (UNCTAD). (2020b). Review of Maritime Transport 2020. United Nations. https:/UnCTAD.org/ system/files/official-document/rmt2020_en.pdf

Wang, Z., He, W., \& Chen, K. (2016). The integrated efficiency of economic development and $\mathrm{CO}_{2}$ emissions among Asia Pacific Economic Cooperation members. Journal of Cleaner Production, 131, 765-772. https://doi. org/10.1016/j.jclepro.2016.04.097

Wasif, Z. M., Shahbaz, M., Hou, F., \& Sinha, A. (2019). From nonrenewable to renewable energy and its impact on economic growth: The role of research \& development expenditures in Asia-Pacific Economic Cooperation countries. Journal of Cleaner Production, (212), 1166-1178. https://doi. org/10.1016/j.jclepro.2018.12.081

World Bank. (2020). World Development Indicators. https://databank.worldbank.org/source/world-development-indicators

World Shipping Council. (2020a). Top 50 World Container Ports [Database]. World Shipping Council Partners Trade. http://www.worldshipping.org/ about-the-industry/global-trade/top-50-world-container-ports 
World Shipping Council. (2020b). Trade Routes. World Shipping Council [Database]. World Shipping Council Partners Trade. http://www.worldshipping. org/about-the-industry/global-trade/trade-routes

$\mathrm{Wu}, \mathrm{Y}$. (2004). Openness, productivity and growth in the APEC economies. Empirical Economics, (29), 593-604. https://doi.org/10.1007/s00181004-0200-1

Wu, Y. C. J., Yuan, C. H., Goh, M., Lu, Y. H. (2016). Regional Port Productivity in APEC. Sustainability, 8(7), 1-17. https://doi.org/10.3390/su8070689

Xu, W., Liu, X., Liu, L., Dore, A. J., Tang, A., Lu, L., Wu, Q., Zhang, Y., Hao, T., Pan, Y., Chen. J., \& Zhang, F. (2019). Impact of emission controls on air quality in Beijing during APEC 2014: Implications from water-soluble ions and carbonaceous aerosol in $\mathrm{PM}_{2.5}$ and their precursors. Atmospheric Environment, (210), 241-252. https://doi.org/10.1016/j.atmosenv.2019.04.050

Yu, Q., Tang, H., Chen, Y., Wu, W., Yang, P., Tang, P., \& Xu, X. (2011, October 19-20). Efficiency Analysis of Agricultural Land Use Based on DEA Method: A Case Study among APEC Economies. International Conference on Computer Distributed Control and Intelligent Environmental Monitoring, Changsha, China, pp. 1216-1219. https://doi.org/10.1109/CDCIEM.2011.565

Zamora, T. A. I. (2017). La eficiencia de las aduanas de la región APEC: Un análisis a través del modelo DEA Malmquist. México y la Cuenca del Pacífico, 6(18), 17-36. https://doi.org/10.32870/mycp.v6i18.554

Zhang, S., Xu, W., Wang, K., Feng, W., Athienitis, A., Hua, G., Okumiya, M., Yoon, G., Cho, D. W., Iyer-Raniga, U., Mazria, E., \& Lyu, Y. (2020). Scenarios of energy reduction potential of zero energy building promotion in the Asia-Pacific region to year 2050. Energy, (213), 1-12. https://doi. org/10.1016/j.energy.2020.118792

Zhou, X., Tan, J., Qin, J., Hu, J., Duan, J., \& Chen, R. (2019). Impact of emissions controls on ambient carbonyls during the Asia-Pacific Economic Cooperation summit in Beijing, China. Environmental Science and Pollution Research, (26), 11875-11887. https://doi.org/10.1007/s11356-01904577-5 